\title{
Revealing - 1 Programmed Ribosomal Frameshifting Mechanisms by Single-Molecule Techniques and Computational Methods
}

\author{
Kai-Chun Chang \\ Institute of Molecular and Cellular Biology, National Taiwan University, Taipei 10617, Taiwan
}

Correspondence should be addressed to Kai-Chun Chang, r00b43003@ntu.edu.tw

Received 25 November 2011; Accepted 16 January 2012

Academic Editor: Shang-Te Danny Hsu

Copyright () 2012 Kai-Chun Chang. This is an open access article distributed under the Creative Commons Attribution License, which permits unrestricted use, distribution, and reproduction in any medium, provided the original work is properly cited.

\begin{abstract}
Programmed ribosomal frameshifting (PRF) serves as an intrinsic translational regulation mechanism employed by some viruses to control the ratio between structural and enzymatic proteins. Most viral mRNAs which use PRF adapt an H-type pseudoknot to stimulate -1 PRF. The relationship between the thermodynamic stability and the frameshifting efficiency of pseudoknots has not been fully understood. Recently, single-molecule force spectroscopy has revealed that the frequency of -1 PRF correlates with the unwinding forces required for disrupting pseudoknots, and that some of the unwinding work dissipates irreversibly due to the torsional restraint of pseudoknots. Complementary to single-molecule techniques, computational modeling provides insights into global motions of the ribosome, whose structural transitions during frameshifting have not yet been elucidated in atomic detail. Taken together, recent advances in biophysical tools may help to develop antiviral therapies that target the ubiquitous -1 PRF mechanism among viruses.
\end{abstract}

\section{Introduction to Programmed - 1 Ribosomal Frameshifting in Viruses}

The genetic information in mRNA is decoded by the ribosome in units of three nucleotides, the codons, being translated into their corresponding amino acids. Consequently, there are three possible reading frames for a given length of nucleotide message. The actual open reading frame starts with the nucleotide triplet AUG and extends with every following triplet being read correctly by the ribosome, ensuring an error rate of frameshifting $<3 \times 10^{-5}$ per codon $[1,2]$. However, programmed ribosomal frameshifting (PRF) is indispensable for many viruses to regulate their protein expression levels from overlapping ORFs. In human immunodeficiency virus type 1 (HIV-1), -1 PRF occurs at a frequency of 5 to $10 \%$ at the junction of $g a g$ and pol genes, resulting in a 20:1 to 10:1 molar ratio of the structural (Gag) to enzymatic proteins (Gag-Pol polyprotein) (Figure 1(a)) [3-6].

The viral -1 PRF site contains three characteristic RNA elements [7-12]. (i) A slippery site with the form of X-XXYYYZ (the dashes separate in-frame triplets), where XXX can be any homopolymeric sequence, YYY can be either AAA or UUU, and $\mathrm{Z}$ can be $\mathrm{A}, \mathrm{U}$ or $\mathrm{C}$. The slippery sequence allows effective base-pairings between the ribosome-bound tRNAs and mRNA even after frameshifting to XXX-YYY (-1 frame). (ii) A 5- to 10-nucleotide-long spacer between the slippery site and the downstream RNA structure. (iii) A downstream pseudoknot or stem-loop (also referred to as a hairpin) structure that is generally thought to act as a "roadblock" to stall the ribosome and subsequently promote ribosome backward slippage.

Most retroviruses adapt pseudoknots as their -1 PRFstimulating elements [13-15]. A typical hairpin- $(\mathrm{H}-)$ type pseudoknot is characterized by base-pairing between a hairpin loop and a single-stranded region outside that loop (Figure 1(b)) $[13,16]$; that is, as shown in Figure 1(b), Stem 2 is formed by base-pairing Loop 1 and the distal end of Loop 2. This brings remote regions of the RNA contour together and gives rise to more complex tertiary interactions, such as base-stacking and triplex base-pairing $[17,18]$. As a result, pseudoknots are generally more stable and efficient in promoting $-1 \mathrm{PRF}$, as compared to their 
stem-loop counterparts with the same compositions of base pairs $[14,17,19]$. Notably, there are still cases like HIV-1 that can utilize simple stem-loops to promote -1 PRF effectively $[19,20]$.

Subtle alterations in -1 PRF elements have been reported to affect viral production dramatically [21-24]. For HIV-1, despite the intrinsically high mutation rate for RNA viruses, Biswas et al. found that of the 1,000 HIV-1 slippery sequences they obtained, all the UUUUUUA slippery heptamers are exactly identical. Substituting this site with another equivalently efficient slippery sequence, namely, UUUAAAA, can reduce viral titer more than 1,000-fold [4, 25]. More recently, annexin A2 (ANXA2), an eukaryotic multifunctional protein, has been shown to bind the pseudoknot of avian coronavirus infectious bronchitis virus (IBV) and reduce the viral -1 PRF efficiency [26]. Accordingly, the authors suspected that ANXA2 might interact more generally with other viral RNA pseudoknots, thereby acting as an antiviral regulator in eukaryotic cells [26].

Despite -1 PRF's ubiquity among infectious viruses, and its promising role for serving as an antiviral target $[3,4,6,27]$, the precise molecular mechanism remains elusive. This paper aims to address how recent developments in biophysical tools, specifically single-molecule techniques and computational modeling, can help to elucidate the mechanochemical basis for -1 PRF.

\section{Single-Molecule Techniques Reveal Mechanochemical Details for Pseudoknot-Stimulated - 1 PRF}

Since the diameter of the mRNA entry tunnel in the ribosome is too small to accommodate the dimensions of doublestranded RNA, any RNA secondary structures must be disrupted before being read by the ribosome $[28,29]$. One may hence expect that the thermodynamic stability of a downstream mRNA structure should correlate with -1 PRF efficiency, as has been observed in the mRNA strands that contain stem-loop stimulatory structures $[19,30]$. Intriguingly, the free-energy change $\Delta G$ of folded and unfolded pseudoknots measured from UV optical melting profiles does not correlate well with the propensity of frameshifting [13, 18, 31-34]. This discrepancy may be attributed to the fact that thermal melting occurs globally at any base pair, but the ribosome can only unwind the duplex sequentially from $5^{\prime}$ to $3^{\prime}$ end of the mRNA [14]. Due to the unique topology of pseudoknots (Figure 1(b)), the downstream Stem 2 causes supercoiling in the Stem 1 via base-pairing as the ribosome attempts to unwind the $5^{\prime}$-end of Stem 1 . Accordingly, Stem 2 must be simultaneously disrupted before allowing ribosome translocation through the entire Stem 1, providing further torsional restraint to the ribosome $[31,35,36]$. In contrast, a simple stem-loop can rotate freely during unwinding. Therefore, other than tertiary interactions, the restriction in rotational freedom explains the superior mechanical stability and -1 PRF efficiency of pseudoknots, compared with energetically equivalent stem-loops $[31,35]$. The total unwinding work exerted by the ribosome would thus be larger than the
$\Delta G$ required for just melting the pseudoknots, with some fraction of work dissipated irreversibly [31]. Then, it can be inferred that -1 PRF efficiency should correlate more with the mechanical force required for "pulling" RNA pseudoknots apart, which resembles successive RNA unwinding by the ribosome $[7,14,31,32]$. Such mechanical pulling of an RNA pseudoknot can be readily carried out by optical tweezers.

An optical trap is formed by focusing a laser beam to the vicinity of a transparent particle that diffracts the incident light $[37,38]$. The particle thus experiences a force from the diffracted photons due to momentum transfer. The intensity profile of the laser beam is chosen to adapt a Gaussian gradient, such that small displacements of the particle $(\sim 150 \mathrm{~nm})$ from the beam center produce a counteracting force toward its equilibrium position, acting like a simple harmonic spring. The spring constant, which is determined through control experiments in advance, depends on both the laser profiles and the dielectric properties of trapped objects [3739].

By monitoring and/or manipulating biomolecules individually, single-molecule techniques are able to unveil stochastic behaviors and rare events that are otherwise hidden in the ensemble averages from a "bulk" biochemical assay ("in bulk" for short). To facilitate single-molecule manipulation on optical tweezers, two DNA handles are attached to micron-sized polystyrene beads through biotin-streptavidin and digoxigenin-antibody interactions, respectively [48]. The RNA molecule of interest, usually a stem-loop or pseudoknot, is flanked by the DNA handles. One of the beads is trapped by a laser beam, while the other is pulled by a micropipette or another trapping laser (Figure 2(a)).

Chen et al. discovered that the unwinding force measured by optical tweezers correlates strongly with the -1 PRF frequency (Figure 2(b)) [32]. Extrapolation of the data predicts that $100 \%-1$ PRF efficiency would be reached by a pseudoknot with an unfolding force around $57 \mathrm{pN}$. The authors reasoned that pseudoknots with unfolding force above $\sim 60 \mathrm{pN}$ would completely stall the ribosome and result in an abortive translation [32]. Although this prediction has not been confirmed directly, such "roadblocking" effect has been observed in bulk recently [7].

Consistent with the torsional restraint model $[35,36]$, a similar experiment conducted by Hansen et al. showed that the work performed by optical tweezers during mechanical unfolding of a IBV-based pseudoknot $(501 \pm 36 \mathrm{~kJ} / \mathrm{mol}$, see PK401 in [31]) is much larger than the theoretically estimated free-energy cost $(292 \mathrm{~kJ} / \mathrm{mol})$ required for both RNA unfolding and stretching [31], with a significant amount of the performed work dissipated irreversibly. The need for extra energy input makes the pseudoknot more resistant to unfolding by optical tweezers and presumably by ribosomes, when compared with its hairpin counterparts with equivalent base-pairing energies. The model also explains the observation that the length and predicted stability of Stem 1 do not always correlate with the frequency of frameshifting,since the effect of torsional restraint must also be taken into consideration; for example, Napthine et al. found that an IBV-derived pseudoknot with $12 \mathrm{bp}$ Stem 1 


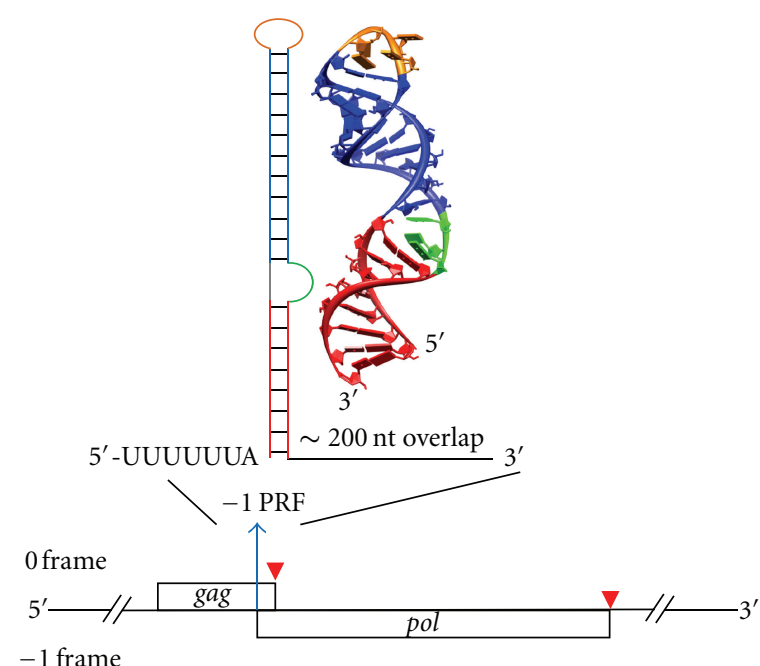

(a)
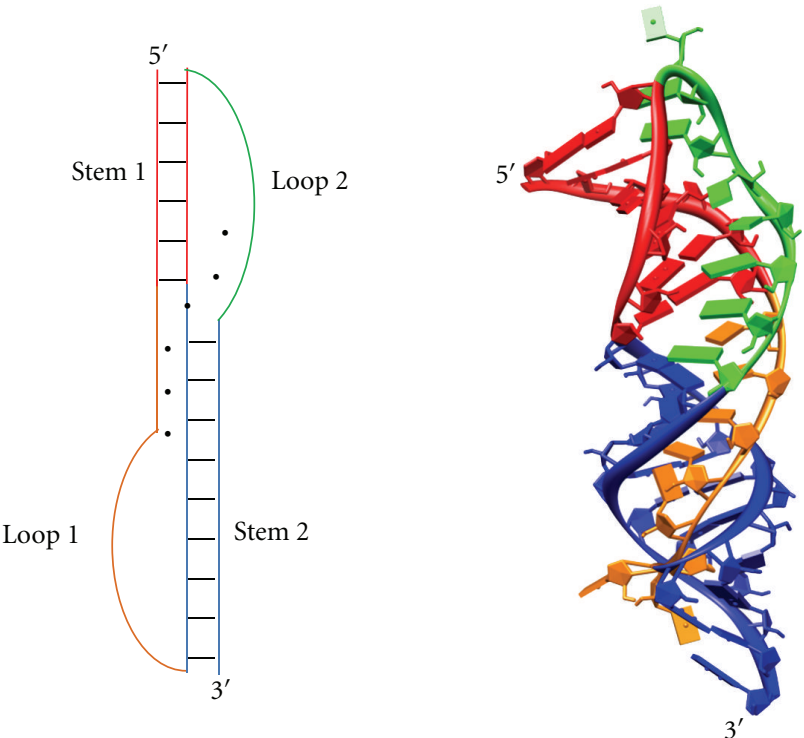

(b)

FIGURE 1: (a) Structure of HIV gag/gag-pol ORFs, with stop codons indicated by red arrow heads. The pol ORF does not contain a start codon and can only be initiated by -1 PRF within the gag ORF. Therefore, the two ORFs are said to be "overlapping" by a length of $\sim 200 \mathrm{nt}$ [3]. The secondary and NMR-resolved three-dimensional structures for the -1 PRF-stimulating stem-loop are illustrated with corresponding colors, whereas base pairs are indicated by short black bars [20,40] (PDB 1Z2J). Stems are shown in red and blue, and loops are shown in orange and green. (b) A minimal ( $\triangle$ U177) human telomerase pseudoknot adapts a canonical H-type pseudoknot configuration [18, 32] (PDB 1YMO). Tertiary major-groove and minor-groove interactions (base triples) are represented by black dots between bases in the secondary structure depiction. The black dot at the junction of Stem 1 and Stem 2 indicates tertiary interaction between Loop 1 (orange) and Loop 2 (green). This RNA structure is not involved in translational regulation, but rather in the activity of the telomerase complex [18, 41]. Its well-known structure makes it an ideal RNA pseudoknot system for studying frameshifting [32, 42]. Stem 1, Loop 1, Stem 2, and Loop 2 are shown in red, orange, blue, and green, respectively. All 3D molecular representations in this paper were produced using the UCSF Chimera package from the Resource for Biocomputing, Visualization, and Informatics at the University of California, San Francisco, USA (supported by NIH P41 RR001081) [43].

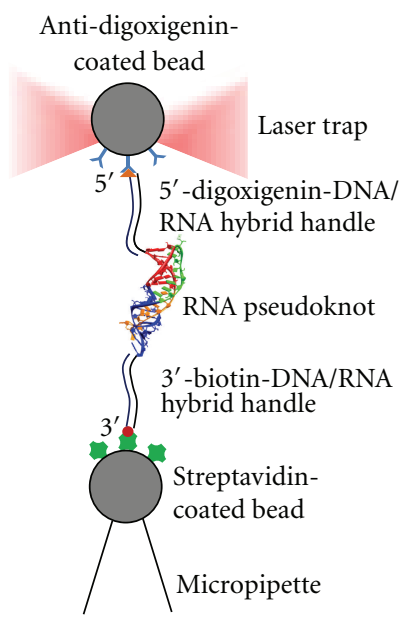

(a)

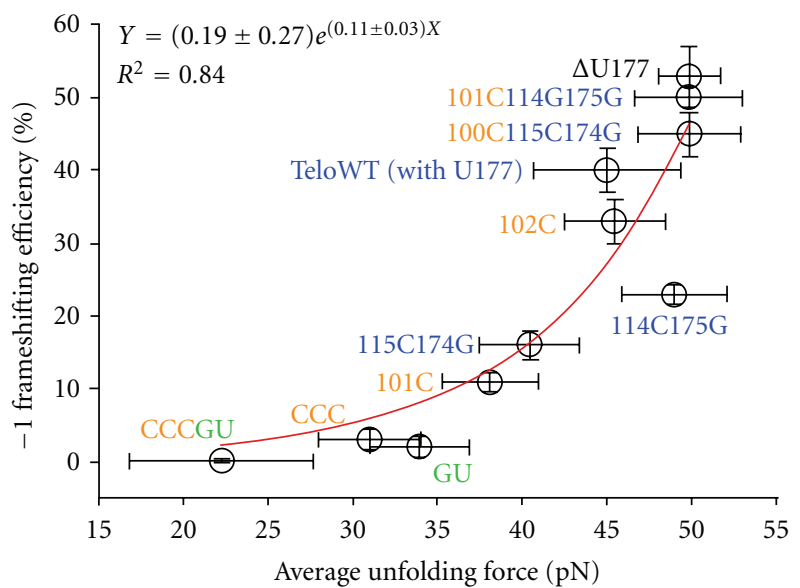

(b)

Figure 2: (a) Schematic representation of optical tweezing. The RNA pseudoknot is flanked by DNA handles that are end-labeled with biotin and digoxigenin, respectively. The handles can then attach to surface-modified beads via biotin-streptavidin and digoxigenin-antibody interactions. Finally, a trapping laser and a micropipette that moves away from the trap produce tension force on the construct. (b) Correlation between -1 PRF efficiencies and externally applied unwinding forces for different pseudoknot constructs. For details of each construct, the reader is referred to reference [32]. Circles indicate average values. Error bars for -1 frameshifting efficiencies along the $y$ axis are standard errors of the means from in vitro bulk frameshifting assay. Error bars for unfolding forces are standard deviations of the optical tweezing measurements. Frameshifting efficiencies correlate better with unfolding forces $\left(R^{2}=0.84\right)$ than melting free energies of pseudoknots $\left(R^{2}=0.59\right.$, see [32] $)$. (b) is reproduced with permission from [32]. 
$\left(\Delta G_{\text {Stem } 1 \text {, theory }}=-67.4 \mathrm{~kJ} / \mathrm{mol}, 55 \%-1 \mathrm{PRF}\right)$ actually stimulates -1 PRF more efficiently than that with a $13 \mathrm{bp}$ Stem $1\left(\Delta G_{\text {Stem } 1, \text { theory }}=-80.0 \mathrm{~kJ} / \mathrm{mol}, 47 \%-1 \mathrm{PRF}\right)[49]$.

Despite the strong mechanical strength of a pseudoknot, HIV-1 is rather unique in that it utilizes a simple stem-loop as its -1 PRF-stimulating element [20], and, as described above, the -1 PRF efficiency is susceptible to mutations in the UUUUUUA frameshifting site (virtually from $\sim 5 \%$ to $0 \%$ for some point mutations) [4]. Therefore, the role of an efficient slippery sequence should also be taken into consideration. Indeed, the slippery nature of a poly $(U)$ template has been demonstrated by optical tweezers previously [50].

While optical tweezers reveal the mechanical properties of mRNAs, single-molecule Förster resonance energy transfer (smFRET) serves as a powerful tool for probing conformational changes of single ribosomes [51-57]. When a laser beam is totally and internally reflected in a microscope, it produces an evanescent wave on the other side of the interface. This evanescent wave decays exponentially with distance, and therefore can only penetrate $\sim 100 \mathrm{~nm}$ into the reaction chamber $[58,59]$. SmFRET exploits this property to excite only the fluorescent samples immobilized on the surface, greatly reducing the background signal from free fluorescent molecules outside the evanescent field. The immobilized fluorescent molecules appear as diffraction-limited spots that can be visualized and recorded by an electron multiplying charge coupled device (EMCCD) camera. The fluorescence intensities and the corresponding FRET efficiencies of single molecules can thus be measured and calculated individually.

A recent smFRET experiment has utilized fluorescently labeled ribosomes to correlate recurring fluctuations in FRET efficiency to intersubunit conformational changes, which in turn indicate ribosomal translocation events [52]. This design allowed the researchers to observe ribosome slipping at codon resolution, which revealed a small fraction $(<2 \%)$ of the ribosome traces exhibiting FRET cycles larger than the mRNA coding length of the homopolymeric poly(U) template. In contrast, additional FRET cycles that are indicative of ribosomal slippage, were not observed when a heteropolymeric template was translated [52]. These results again confirm the notion that the $\mathrm{U}$-rich sequence is very slippery [25].

Although a recent study suggests that stem-loops can serve as efficient - 1 PRF stimulators, the experiment has utilized a slippery sequence, UUUAAAC, that appears to be even more efficient in promoting -1 PRF than the HIV-1 UUUUUUA motif (41.7\% versus $24.7 \%$ when placed upstream of a minimal IBV pseudoknot) $[19,25]$. Taken together, albeit a pseudoknot structure provides a stronger mechanical hindrance for ribosome progression, an efficient slippery sequence probably works synergistically with a less topologically restrained stem-loop. However, the quantitative relationships between these components, as to why certain sequences are many folds more slippery than others [25], require further investigations.

The above examples demonstrate that single-molecule spectroscopy can straightforwardly access physical quantities that seem to better correlate with pseudoknot-stimulated
-1 PRF efficiencies, thereby providing unprecedented mechanochemical details for the underlying mechanisms $[16,31$, 32].

\section{Coarse-Grained Elastic Network Model Provides Further Insights into Global Motions of the Ribosome and May Guide the Labeling Scheme for Single-Molecule Spectroscopy}

The ribosome has been shown to possess intrinsic mRNA helicase activity for resolving duplex structures of the mRNA during translation in vitro [29]. Interestingly, in addition to the superior stability of pseudoknots arising from tertiary interactions and torsional restraints, it has been proposed that stereochemical mismatch between the pseudoknot structure and the geometry of the putative ribosomal helicase sites would block the entry of downstream mRNA [28, 44]. This view is supported by the fact that some mutations in Loop 2 that are not anticipated to affect tertiary interactions, but are suspected to alter contacts with the ribosome, significantly lower - 1 PRF efficiencies [60]. This gives rise to the interesting possibility that other than acting as a general mechanical hindrance, specific interactions between the pseudoknot and the mRNA entry site of the ribosome could promote -1 PRF by inducing conformational changes of the ribosome complex allosterically. Indeed, although both pseudoknots and stem-loops can stall ribosomes, only pseudoknots are able to induce distortion in the P-site tRNA, as observed in the cryoelectron microscopy (cryo-EM) maps of stalled mammalian $80 \mathrm{~S}$ ribosomes (Figure 3(a)) [44]. The low resolution (16.2 $\AA$ ), however, was not sufficient for visualizing atomic details of the structural dynamics during frameshifting. Unfortunately, high-resolution single-molecule techniques are also constrained by their pulling or labeling sites and are consequently blind to such global structural changes. Computational modeling can, on the other hand, monitor overall motions of the molecule from detailed structural models.

A coarse-grained elastic network model [61] only takes the $\mathrm{C}_{\alpha}$ atom for each amino acid residue into account as "nodes" in a protein network [62]. For each nucleotide, 2 or 3 representative atoms are assigned as nodes $[63,64]$. The interaction between each node is then approximated by simple harmonic potential, which dictates the fluctuations of these nodes. The nodal vibrations are further decomposed into a number of normal modes, whose contributions to the overall dynamics of a protein are inversely weighted by their respective frequency-squared. As a consequence, the biologically relevant motions of a protein, which generally involve large-scale conformational changes, are dominated by lowfrequency modes in ENM [65-67].

ENM is based on the view that protein dynamics is largely determined by the topology of native contacts, as has been proposed and supported by several studies [68-72]. Accordingly, the physical and chemical properties of each residue are not taken into consideration in ENM, and the computational complexity is greatly reduced compared with 


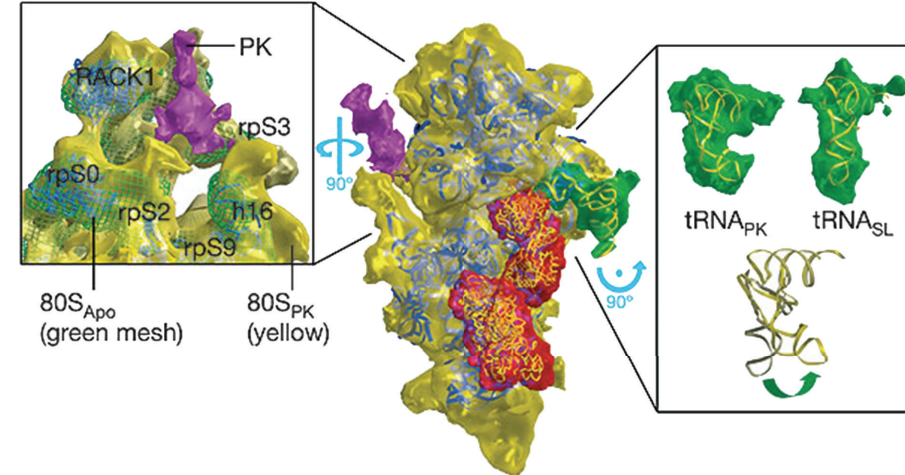

(a)

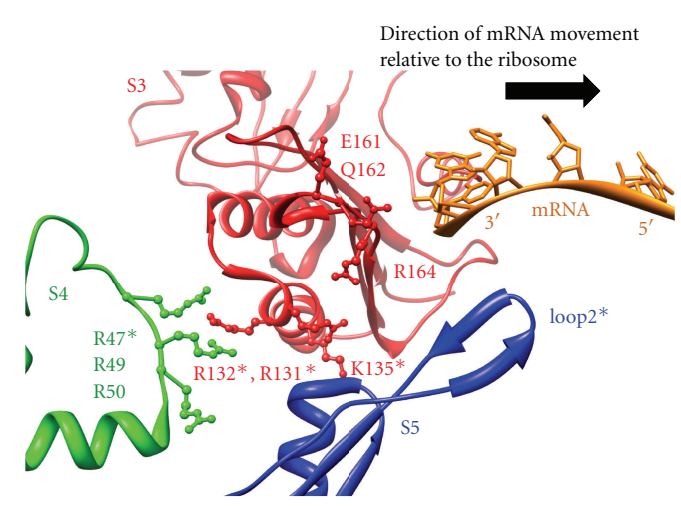

(b)

Figure 3: (a) Cryo-EM reconstituted map of pseudoknot-stalled mammalian 40S small ribosomal subunit. The close-up view on the left shows the pseudoknot (PK, purple) binding to the putative ribosomal helicase site, namely, rpS3 (equivalent to prokaryotic S3), rpS9 (S4 in prokaryotes), and $\mathrm{rpS} 2$ (S5 in prokaryotes). Compared with the vacant $80 \mathrm{~S}$ ribosome map ( $80 \mathrm{~S}_{\mathrm{Apo}}$, green mesh), these subunits lining the mRNA entrance tunnel (yellow) move slightly toward the pseudoknot. The close-up view on the right shows pseudoknot-stalled P-site

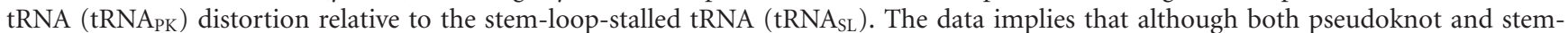
loop promote ribosome stalling, only pseudoknot can induce conformational changes in the ribosome complex. Figure reproduced with permission from [44]. (b) Close-up view of prokaryotic mRNA entrance tunnel [45] (PDB 3KNJ). Viewed from interior of the ribosome, residues implicated in interacting with the mRNA by ENM are labeled [46], and shown in ball-and-stick representations. Asterisks denote functional residues reported to be involved in ribosomal helicase activity (residues in S3 and S4) and translational fidelity (loop 2 in S5) by previous experiments $[29,47]$. The ribosome reads mRNA in a $5^{\prime}$ to $3^{\prime}$ fashion, that is, opposite to mRNA movement indicated by the black arrow. Messenger RNA, S3, S4, and S5 are shown in orange, red, green, and blue, respectively.

molecular dynamics simulations [71, 73]. It takes even less time to calculate only the low-frequency modes that dominate macromolecular motions, making ENM especially suitable for modeling large complexes [70]. Indeed, the ratchetlike motion of the ribosome, one of the most important global motions characterizing ribosomal translocation [74], was clearly described by ENM calculations that only took a few slow modes into account $[64,71]$. Furthermore, global deformations of the ribosome can be calculated by ENM iteratively based on X-ray structures and then fitted into cryoEM structures originated from different states of the ribosome bound with various factors, unraveling large-scale conformational changes from inherently low-resolution cryoEM images [75].

The relationship between accommodation of A-site aminoacyl-tRNA and dissociation of E-site deacylated tRNA remained elusive for years $[16,76]$. Based on the ENM simulation results, Wang et al. predicted that the movements of A-site and E-site tRNAs are uncoupled (the orientation correlation is 0.165 , where a unity value would indicate perfectly concerted motions) [64]. This prediction has been supported by correlation analysis of a later single-molecule fluorescence experiment (correlation coefficient $r=0.04$ ) [76]. Likewise, Kurkcuoglu et al. utilized the same approach to detect possible active sites responsible for the intrinsic helicase activity of the ribosome (Figure 3(b)) [46]. The predictions not only agreed with previous results [29], but also gave more possible key residues that have not been confirmed experimentally. Therefore, ENM, which samples equilibrium dynamics and predicts global conformational changes, can guide the labeling scheme for future single-molecule experiments crucial for probing direct interactions between the ribosomal helicase and the pseudoknot.

Modeling perturbed ribosomal dynamics induced by pseudoknots can be readily carried out with ENM and linear response theories [77], where the magnitude of perturbation force exerted on the ribosome can be inferred from unwinding forces of pseudoknots provided by single-molecule measurements. Alternatively, the pulling force directly applied on the ribosome by optical tweezers provides another plausible experimental approach for model validations and refinement. This scheme may as well be applied to other supramolecular assemblages.

\section{Future Perspectives}

Due to the asynchronous nature of stochastic reactions, detailed molecular mechanisms are often difficult to be inferred from conventional bulk experiments. Nevertheless, developments of recent biophysical tools, particularly singlemolecule techniques, have elucidated much about the mechanical and functional properties of pseudoknots [31, 32, $41,44]$, the action of the ribosomal helicase $[46,78]$, and the mechanisms of translational machinery as a whole [52, 76, 79, 80]. Indeed, combining data from cryo-EM, X-ray crystallography, as well as molecular dynamics simulations/ modeling has provided predictions and insights into the interactions between the ribosomal L1 stalk and tRNAs, which agree well with smFRET results [80]. It is tempting to ask what we can learn by applying similarly combined methodologies to investigations of -1 PRF mechanisms. 
Structural, computational, and single-molecule approaches are complementary to each other. Computational modeling and simulations unravel the dynamic nature of molecules and provide physics-based methods for protein deformations in the presence of externally applied forces $[69,73,81,82]$. The calculations could make "rational" biotin/digoxigenin labeling possible in a single-molecule experiment, by labeling the ribosome at sites that cause the least conformational changes and the resulting dissociation from the mRNA during optical tweezing. The mechanical descriptions thereof can be rather physiologically relevant, as in the case of titin kinase, which serves as a molecular force sensor in muscle cells [81-83]. On the other hand, single-molecule force and fluorescence spectroscopies probe real-time structural transitions, such as folding-unfolding as well as stretching, for proteins [84-86], RNAs [87], and complexes [41, 88]. These data can be subsequently used to validate and/or refine the physics models and molecular simulations.

Understandings to the translational machinery as well as -1 PRF mechanisms have suggested attractive targets for antiviral therapies. Although it is possible to develop drugs that target the eukaryotic $80 \mathrm{~S}$ ribosome and alter -1 PRF $[89,90]$, the side-effects are unclear, owing to potential cellular genes that utilize frameshifting, but have not yet been found [91-93]. Developing drugs that specifically interact with viral -1 PRF-stimulating structures could be a good intervention strategy. Indeed, small ligands have been identified to alter viral -1 PRF efficiencies by binding to the -1 PRF-promoting stem-loop in HIV-1 and the pseudoknot in severe acute respiratory syndrome coronavirus (SARS-CoV) $[6,21,27]$. As described above, single-molecule force spectroscopy can provide the unfolding forces of various RNA structures, which correlate with -1 PRF efficiencies much better than thermodynamic stabilities. Studies with various mutant -1 PRF-promoting structures may facilitate drug discovery by identifying the essential residues and bondings responsible for their mechanical stabilities as well as interactions with the ribosome. Accordingly, combining the new biophysical tools sheds light on how future antiviral agents can be developed to work against the ubiquitous -1 PRF mechanisms among viruses.

\section{Acknowledgments}

The author gratefully thanks Dr. Jin-Der Wen of the Institute of Molecular and Cellular Biology (IMCB), National Taiwan University, and Dr. Lee-Wei Yang of Institute of the Bioinformatics and Structural Biology, National Tsing-Hua University for their enlightenment and support for this paper. He thanks Dr. Robert Anderson of Department of Microbiology and Immunology, Dalhousie University, Canada, for critical reading of this paper. He would also like to acknowledge National Science Council and IMCB for their financial support of this work and related research.

\section{References}

[1] J. F. Curran and M. Yarus, "Base substitutions in the tRNA anticodon arm do not degrade the accuracy of reading frame maintenance," Proceedings of the National Academy of Sciences of the United States of America, vol. 83, no. 17, pp. 6538-6542, 1986.

[2] J. F. Atkins, D. Elseviers, and L. Gorini, "Low activity of - galactosidase in frameshift mutants of Escherichia coli," Proceedings of the National Academy of Sciences of the United States of America, vol. 69, no. 5, pp. 1192-1195, 1972.

[3] T. Jacks, M. D. Power, F. R. Masiarz, P. A. Luciw, P. J. Barr, and H. E. Varmus, "Characterization of ribosomal frameshifting in HIV-1 gag-pol expression,” Nature, vol. 331, no. 6153, pp. 280-283, 1988.

[4] P. Biswas, X. Jiang, A. L. Pacchia, J. P. Dougherty, and S. W. Peltz, "The Human Immunodeficiency Virus Type 1 Ribosomal Frameshifting Site Is an Invariant Sequence Determinant and an Important Target for Antiviral Therapy," Journal of Virology, vol. 78, no. 4, pp. 2082-2087, 2004.

[5] Y. N. Vaishnav and F. Wong-Staal, "The biochemistry of AIDS," Annual Review of Biochemistry, vol. 60, pp. 577-630, 1991.

[6] R. J. Marcheschi, M. Tonelli, A. Kumar, and S. E. Butcher, "Structure of the HIV-1 frameshift site RNA bound to a small molecule inhibitor of viral replication," ACS Chemical Biology, vol. 6, no. 8, pp. 857-864, 2011.

[7] J. Tholstrup, L. B. Oddershede, and M. A. Sørensen, "MRNA pseudoknot structures can act as ribosomal roadblocks," Nucleic Acids Research, vol. 40, no. 1, pp. 303-313, 2012.

[8] P. J. Farabaugh, "Programmed translational frameshifting," Microbiological Reviews, vol. 60, no. 1, pp. 103-134, 1996.

[9] J. D. Dinman, T. Icho, and R. B. Wickner, "A - 1 ribosomal frameshift in a double-stranded RNA virus of yeast forms a gag-pol fusion protein," Proceedings of the National Academy of Sciences of the United States of America, vol. 88, no. 1, pp. 174-178, 1991.

[10] E. B. Dam, C. W. A. Pleij, and L. Bosch, "RNA pseudoknots: translational frameshifting and readthrough on viral RNAs," Virus Genes, vol. 4, no. 2, pp. 121-136, 1990.

[11] I. Brierley, A. J. Jenner, and S. C. Inglis, "Mutational analysis of the 'slippery-sequence' component of a coronavirus ribosomal frameshifting signal," Journal of Molecular Biology, vol. 227, no. 2, pp. 463-479, 1992.

[12] T. Jacks, H. D. Madhani, F. R. Masiarz, and H. E. Varmus, "Signals for ribosomal frameshifting in the rous sarcoma virus gag-pol region," Cell, vol. 55, no. 3, pp. 447-458, 1988.

[13] D. P. Giedroc, C. A. Theimer, and P. L. Nixon, "Structure, stability and function of RNA pseudoknots involved in stimulating ribosomal frameshifting," Journal of Molecular Biology, vol. 298, no. 2, pp. 167-185, 2000.

[14] L. Green, C. H. Kim, C. Bustamante, and I. Tinoco, "Characterization of the Mechanical Unfolding of RNA Pseudoknots," Journal of Molecular Biology, vol. 375, no. 2, pp. 511-528, 2008.

[15] I. Brierley and S. Pennell, "Structure and function of the stimulatory RNAs involved in programmed eukaryotic -1 ribosomal frameshifting," Cold Spring Harbor Symposia on Quantitative Biology, vol. 66, pp. 233-248, 2001.

[16] D. P. Giedroc and P. V. Cornish, "Frameshifting RNA pseudoknots: structure and mechanism," Virus Research, vol. 139, no. 2, pp. 193-208, 2009.

[17] P. L. Nixon and D. P. Giedroc, "Energetics of a strongly $\mathrm{pH}$ dependent RNA tertiary structure a frameshifting pseudoknot," Journal of Molecular Biology, vol. 296, no. 2, pp. 659$671,2000$.

[18] C. A. Theimer, C. A. Blois, and J. Feigon, "Structure of the human telomerase RNA pseudoknot reveals conserved tertiary 
interactions essential for function," Molecular Cell, vol. 17, no. 5, pp. 671-682, 2005.

[19] C.-H. Yu, M. H. Noteborn, C. W. A. Pleij, and R. C. L. Olsthoorn, "Stem-loop structures can effectively substitute for an RNA pseudoknot in -1 ribosomal frameshifting," Nucleic Acids Research, vol. 39, no. 20, pp. 8952-8959, 2011.

[20] D. W. Staple and S. E. Butcher, "Solution structure and thermodynamic investigation of the HIV-1 frameshift inducing element," Journal of Molecular Biology, vol. 349, no. 5, pp. 1011-1023, 2005.

[21] M. Hung, P. Patel, S. Davis, and S. R. Green, "Importance of ribosomal frameshifting for human immunodeficiency virus type 1 particle assembly and replication," Journal of Virology, vol. 72, no. 6, pp. 4819-4824, 1998.

[22] D. Dulude, Y. A. Berchiche, K. Gendron, L. Brakier-Gingras, and N. Heveker, "Decreasing the frameshift efficiency translates into an equivalent reduction of the replication of the human immunodeficiency virus type 1," Virology, vol. 345, no. 1, pp. 127-136, 2006.

[23] M. Shehu-Xhilaga, S. M. Crowe, and J. Mak, "Maintenance of the Gag/Gag-Pol ratio is important for human immunodeficiency virus type 1 RNA dimerization and viral infectivity," Journal of Virology, vol. 75, no. 4, pp. 1834-1841, 2001.

[24] I. Brierley and F. J. dos Ramos, "Programmed ribosomal frameshifting in HIV-1 and the SARS-CoV," Virus Research, vol. 119, no. 1, pp. 29-42, 2006.

[25] I. Brierley, A. J. Jenner, and S. C. Inglis, "Mutational analysis of the 'slippery-sequence' component of a coronavirus ribosomal frameshifting signal," Journal of Molecular Biology, vol. 227, no. 2, pp. 463-479, 1992.

[26] H. Kwak, M. W. Park, and S. Jeong, "Annexin A2 binds RNA and reduces the frameshifting efficiency of Infectious Bronchitis Virus," PLoS ONE, vol. 6, no. 8, 2011.

[27] S.-J. Park, Y.-G. Kim, and H.-J. Park, "Identification of rna pseudoknot-binding ligand that inhibits the -1 ribosomal frameshifting of SARS-coronavirus by structure-based virtual screening," Journal of the American Chemical Society, vol. 133, no. 26, pp. 10094-10100, 2011.

[28] G. Z. Yusupova, M. M. Yusupov, J. H. D. Cate, and H. F. Noller, "The path of messenger RNA through the ribosome," Cell, vol. 106, no. 2, pp. 233-241, 2001.

[29] S. Takyar, R. P. Hickerson, and H. F. Noller, "mRNA helicase activity of the ribosome," Cell, vol. 120, no. 1, pp. 49-58, 2005.

[30] L. Bidou, G. Stahl, B. Grima, H. Liu, M. Cassan, and J. P. Rousset, "In vivo HIV-1 frameshifting efficiency is directly related to the stability of the stem-loop stimulatory signal," RNA, vol. 3, no. 10, pp. 1153-1158, 1997.

[31] T. M. Hansen, S. Nader S Reihani, L. B. Oddershede, and M. A. Sørensen, "Correlation between mechanical strength of messenger RNA pseudoknots and ribosomal frameshifting," Proceedings of the National Academy of Sciences of the United States of America, vol. 104, no. 14, pp. 5830-5835, 2007.

[32] G. Chen, K. Y. Chang, M. Y. Chou, C. Bustamante, and I. Tinoco, "Triplex structures in an RNA pseudoknot enhance mechanical stability and increase efficiency of -1 ribosomal frameshifting," Proceedings of the National Academy of Sciences of the United States of America, vol. 106, no. 31, pp. 1270612711, 2009.

[33] X. Chen, M. Chamorro, S. I. Lee et al., "Structural and functional studies of retroviral RNA pseudoknots involved in ribosomal frameshifting: nucleotides at the junction of the two stems are important for efficient ribosomal frameshifting," EMBO Journal, vol. 14, no. 4, pp. 842-852, 1995.
[34] C. A. Theimer and D. P. Giedroc, "Contribution of the intercalated adenosine at the helical junction to the stability of the gag-pro frameshifting pseudoknot from mouse mammary tumor virus," RNA, vol. 6, no. 3, pp. 409-421, 2000.

[35] E. P. Plant and J. D. Dinman, "Torsional restraint: a new twist on frameshifting pseudoknots," Nucleic Acids Research, vol. 33, no. 6, pp. 1825-1833, 2005.

[36] J. D. Dinman, "Ribosomal frameshifting in yeast viruses," Yeast, vol. 11, no. 12, pp. 1115-1127, 1995.

[37] K. C. Neuman and S. M. Block, "Optical trapping," Review of Scientific Instruments, vol. 75, no. 9, pp. 2787-2809, 2004.

[38] K. C. Neuman and A. Nagy, "Single-molecule force spectroscopy: optical tweezers, magnetic tweezers and atomic force microscopy," Nature Methods, vol. 5, no. 6, pp. 491-505, 2008.

[39] W. J. Greenleaf, M. T. Woodside, and S. M. Block, "Highresolution, single-molecule measurements of biomolecular motion," Annual Review of Biophysics and Biomolecular Structure, vol. 36, pp. 171-190, 2007.

[40] D. Dulude, M. Baril, and L. Brakier-Gingras, "Characterization of the frameshift stimulatory signal controlling a programmed -1 ribosomal frameshift in the human immunodeficiency virus type 1," Nucleic Acids Research, vol. 30, no. 23, pp. 5094-5102, 2002.

[41] M. Mihalusova, J. Y. Wu, and X. Zhuang, "Functional importance of telomerase pseudoknot revealed by single-molecule analysis," Proceedings of the National Academy of Sciences of the United States of America, vol. 108, no. 51, pp. 20339-20344, 2011.

[42] G. Chen, J. D. Wen, and I. Tinoco, "Single-molecule mechanical unfolding and folding of a pseudoknot in human telomerase RNA," RNA, vol. 13, no. 12, pp. 2175-2188, 2007.

[43] E. F. Pettersen, T. D. Goddard, C. C. Huang et al., "UCSF Chimera-a visualization system for exploratory research and analysis," Journal of Computational Chemistry, vol. 25, no. 13, pp. 1605-1612, 2004.

[44] O. Namy, S. J. Moran, D. I. Stuart, R. J. C. Gilbert, and I. Brierley, "A mechanical explanation of RNA pseudoknot function in programmed ribosomal frameshifting," Nature, vol. 441, no. 7090, pp. 244-247, 2006.

[45] R. E. Stanley, G. Blaha, R. L. Grodzicki, M. D. Strickler, and T. A. Steitz, "The structures of the anti-tuberculosis antibiotics viomycin and capreomycin bound to the $70 \mathrm{~S}$ ribosome," $\mathrm{Na}$ ture Structural and Molecular Biology, vol. 17, no. 3, pp. 289293, 2010.

[46] O. Kurkcuoglu, P. Doruker, T. Z. Sen, A. Kloczkowski, and R. L. Jernigan, "The ribosome structure controls and directs mRNA entry, translocation and exit dynamics," Physical Biology, vol. 5, no. 4, Article ID 046005, 2008.

[47] N. Kirthi, B. Roy-Chaudhuri, T. Kelley, and G. M. Culver, "A novel single amino acid change in small subunit ribosomal protein S5 has profound effects on translational fidelity," RNA, vol. 12, no. 12, pp. 2080-2091, 2006.

[48] J. Liphardt, B. Onoa, S. B. Smith, Tinoco, and C. Bustamante, "Reversible unfolding of single RNA molecules by mechanical force," Science, vol. 292, no. 5517, pp. 733-737, 2001.

[49] S. Napthine, J. Liphardt, A. Bloys, S. Routledge, and I. Brierley, "The role of RNA pseudoknot stem 1 length in the promotion of efficient - 1 ribosomal frameshifting," Journal of Molecular Biology, vol. 288, no. 3, pp. 305-320, 1999.

[50] F. Vanzi, Y. Takagi, H. Shuman, B. S. Cooperman, and Y. E. Goldman, "Mechanical studies of single ribosome/mRNA complexes," Biophysical Journal, vol. 89, no. 3, pp. 1909-1919, 2005.

[51] P. V. Cornish, D. N. Ermolenko, D. W. Staple et al., "Following movement of the L1 stalk between three functional states 
in single ribosomes," Proceedings of the National Academy of Sciences of the United States of America, vol. 106, no. 8, pp. 2571-2576, 2009.

[52] C. E. Aitken and J. D. Puglisi, "Following the intersubunit conformation of the ribosome during translation in real time," Nature Structural and Molecular Biology, vol. 17, no. 7, pp. 793-800, 2010.

[53] J. Y. Fei, A. C. Richard, J. E. Bronson, and R. L. Gonzalez Jr, "Transfer RNA-mediated regulation of ribosome dynamics during protein synthesis," Nature Structural and Molecular Biology, vol. 18, no. 9, pp. U1043-U1051, 2011.

[54] J. Fei, P. Kosuri, D. D. MacDougall, and R. L. Gonzalez, "Coupling of Ribosomal L1 Stalk and tRNA Dynamics during Translation Elongation," Molecular Cell, vol. 30, no. 3, pp. 348359, 2008.

[55] J. Fei, J. E. Bronson, J. M. Hofman, R. L. Srinivas, C. H. Wiggins, and R. L. Gonzalez, "Allosteric collaboration between elongation factor $\mathrm{G}$ and the ribosomal L1 stalk directs tRNA movements during translation," Proceedings of the National Academy of Sciences of the United States of America, vol. 106, no. 37, pp. 15702-15707, 2009.

[56] J. B. Munro, R. B. Altman, C. S. Tung, J. H. D. Cate, K. Y. Sanbonmatsu, and S. C. Blanchard, "Spontaneous formation of the unlocked state of the ribosome is a multistep process," Proceedings of the National Academy of Sciences of the United States of America, vol. 107, no. 2, pp. 709-714, 2010.

[57] S. H. Sternberg, J. Fei, N. Prywes, K. A. McGrath, and R. L. Gonzalez, "Translation factors direct intrinsic ribosome dynamics during translation termination and ribosome recycling," Nature Structural and Molecular Biology, vol. 16, no. 8, pp. 861-868, 2009.

[58] R. Roy, S. Hohng, and T. Ha, "A practical guide to singlemolecule FRET," Nature Methods, vol. 5, no. 6, pp. 507-516, 2008.

[59] A. Sarkar, R. B. Robertson, and J. M. Fernandez, "Simultaneous atomic force microscope and fluorescence measurements of protein unfolding using a calibrated evanescent wave," Proceedings of the National Academy of Sciences of the United States of America, vol. 101, no. 35, pp. 12882-12886, 2004.

[60] Y. G. Kim, L. Su, S. Maas, A. O’Neill, and A. Rich, “Specific mutations in a viral RNA pseudoknot drastically change ribosomal frameshifting efficiency," Proceedings of the National Academy of Sciences of the United States of America, vol. 96, no. 25, pp. 14234-14239, 1999.

[61] N. Dolzhanskaya, G. Merz, and R. B. Denman, "Alternative splicing modulates protein arginine methyltransferasedependent methylation of fragile X syndrome mental retardation protein," Biochemistry, vol. 45, no. 34, pp. 10385-10393, 2006.

[62] M. Levitt, C. Sander, and P. S. Stern, "Protein normal-mode dynamics: trypsin inhibitor, crambin, ribonuclease and lysozyme," Journal of Molecular Biology, vol. 181, no. 3, pp. 423447, 1985.

[63] M. Delarue and Y. H. Sanejouand, "Simplified normal mode analysis of conformational transitions in DNA-dependent polymerases: the Elastic Network Model," Journal of Molecular Biology, vol. 320, no. 5, pp. 1011-1024, 2002.

[64] Y. Wang, A. J. Rader, I. Bahar, and R. L. Jernigan, "Global ribosome motions revealed with elastic network model," Journal of Structural Biology, vol. 147, no. 3, pp. 302-314, 2004.

[65] B. Brooks and M. Karplus, "Harmonic dynamics of proteins: normal modes and fluctuations in bovine pancreatic trypsin inhibitor," Proceedings of the National Academy of Sciences of the United States of America, vol. 80, no. 21 I, pp. 6571-6575, 1983.

[66] I. Bahar, A. R. Atilgan, M. C. Demirel, and B. Erman, "Vibrational dynamics of folded proteins: significance of slow and fast motions in relation to function and stability," Physical Review Letters, vol. 80, no. 12, pp. 2733-2736, 1998.

[67] T. Haliloglu, I. Bahar, and B. Erman, "Gaussian dynamics of folded proteins," Physical Review Letters, vol. 79, no. 16, pp. 3090-3093, 1997.

[68] K. W. Plaxco, S. Larson, I. Ruczinski et al., "Evolutionary conservation in protein folding kinetics," Journal of Molecular Biology, vol. 298, no. 2, pp. 303-312, 2000.

[69] D. K. Klimov and D. Thirumalai, "Native topology determines force-induced unfolding pathways in globular proteins," Proceedings of the National Academy of Sciences of the United States of America, vol. 97, no. 13, pp. 7254-7259, 2000.

[70] I. Bahar, T. R. Lezon, L. W. Yang, and E. Eyal, "Global dynamics of proteins: bridging between structure and function," Annual Review of Biophysics, vol. 39, no. 1, pp. 23-42, 2010.

[71] F. Tama, M. Valle, J. Frankt, and C. L. Brooks, "Dynamic reorganization of the functionally active ribosome explored by normal mode analysis and cryo-electron microscopy," Proceedings of the National Academy of Sciences of the United States of America, vol. 100, no. 16, pp. 9319-9323, 2003.

[72] I. Bahar, T. R. Lezon, A. Bakan, and I. H. Shrivastava, "Normal mode analysis of biomolecular structures: functional mechanisms of membrane proteins," Chemical Reviews, vol. 110, no. 3, pp. 1463-1497, 2010.

[73] E. Eyal and I. Bahar, "Toward a molecular understanding of the anisotropic response of proteins to external forces: insights from elastic network models," Biophysical Journal, vol. 94, no. 9, pp. 3424-3435, 2008.

[74] J. Frank and R. K. Agrawal, "A ratchet-like inter-subunit reorganization of the ribosome during translocation," Nature, vol. 406, no. 6793, pp. 318-322, 2000.

[75] A. Matsumoto and H. Ishida, "Global Conformational Changes of Ribosome Observed by Normal Mode Fitting for 3D Cryo-EM Structures," Structure, vol. 17, no. 12, pp. 16051613, 2009.

[76] S. Uemura, C. E. Aitken, J. Korlach, B. A. Flusberg, S. W. Turner, and J. D. Puglisi, "Real-time tRNA transit on single translating ribosomes at codon resolution," Nature, vol. 464, no. 7291, pp. 1012-1017, 2010.

[77] A. Kidera, M. Ikeguchi, J. Ueno, and M. Sato, "Protein structural change upon ligand binding: linear response theory," Physical Review Letters, vol. 94, no. 7, Article ID 078102, 2005.

[78] X. Qu, J.-D. Wen, L. Lancaster, H. F. Noller, C. Bustamante, and I. Tinoco, "The ribosome uses two active mechanisms to unwind messenger RNA during translation," Nature, vol. 475, no. 7354, pp. 118-121, 2011.

[79] J.-D. Wen, L. Lancaster, C. Hodges et al., "Following translation by single ribosomes one codon at a time," Nature, vol. 452, no. 7187, pp. 598-603, 2008.

[80] L. G. Trabuco, E. Schreiner, J. Eargle et al., "The Role of L1 Stalk-tRNA Interaction in the Ribosome Elongation Cycle," Journal of Molecular Biology, vol. 402, no. 4, pp. 741-760, 2010.

[81] F. Grater, J. Shen, H. Jiang, M. Gautel, and H. Grubmüller, "Mechanically induced titin kinase activation studied by forceprobe molecular dynamics simulations," Biophysical Journal, vol. 88, no. 2, pp. 790-804, 2005.

[82] E. M. Puchner, A. Alexandrovich, L. K. Ay et al., "Mechanoenzymatics of titin kinase," Proceedings of the National Academy of Sciences of the United States of America, vol. 105, no. 36, pp. 13385-13390, 2008. 
[83] S. Lange, F. Xiang, A. Yakovenko et al., "Cell biology: the kinase domain of titin controls muscle gene expression and protein turnover," Science, vol. 308, no. 5728, pp. 1599-1603, 2005.

[84] M. S. Z. Kellermayer, S. B. Smith, H. L. Granzier, and C. Bustamante, "Folding-unfolding transitions in single titin molecules characterized with laser tweezers," Science, vol. 276, no. 5315, pp. 1112-1116, 1997.

[85] E. A. Shank, C. Cecconi, J. W. Dill, S. Marqusee, and C. Bustamante, "The folding cooperativity of a protein is controlled by its chain topology," Nature, vol. 465, no. 7298, pp. 637640, 2010.

[86] C. M. Kaiser, D. H. Goldman, J. D. Chodera, I. Tinoco Jr, and C. Bustamante, "The ribosome modulates nascent protein folding," Science, vol. 334, no. 6063, pp. 1723-1727, 2011.

[87] M. Steiner, K. S. Karunatilaka, R. K. O. Sigel, and D. Rueda, "Single-molecule studies of group II intron ribozymes," Proceedings of the National Academy of Sciences of the United States of America, vol. 105, no. 37, pp. 13853-13858, 2008.

[88] P. Mangeol, T. Bizebard, C. Chiaruttini, M. Dreyfus, M. Springer, and U. Bockelmann, "Probing ribosomal proteinRNA interactions with an external force," Proceedings of the National Academy of Sciences of the United States of America, vol. 108, no. 45, pp. 18272-18276, 2011.

[89] T. G. Kinzy, J. W. Harger, A. Carr-Schmid et al., "New targets for antivirals: the ribosomal A-site and the factors that interact with it," Virology, vol. 300, no. 1, pp. 60-70, 2002.

[90] J. D. Dinman, M. J. Ruiz-Echevarria, K. Czaplinski, and S. W. Peltz, "Peptidyl-transferase inhibitors have antiviral properties by altering programmed -1 ribosomal frameshifting efficiencies: development of model systems," Proceedings of the $\mathrm{Na}$ tional Academy of Sciences of the United States of America, vol. 94, no. 13, pp. 6606-6611, 1997.

[91] E. Manktelow, K. Shigemoto, and I. Brierley, "Characterization of the frameshift signal of Edr, a mammalian example of programmed -1 ribosomal frameshifting," Nucleic Acids Research, vol. 33, no. 5, pp. 1553-1563, 2005.

[92] N. M. Wills, B. Moore, A. Hammer, R. F. Gesteland, and J. F. Atkins, "A functional -1 ribosomal frameshift signal in the human paraneoplastic Ma3 gene," Journal of Biological Chemistry, vol. 281, no. 11, pp. 7082-7088, 2006.

[93] M. B. Clark, M. Jänicke, U. Gottesbühren et al., "Mammalian gene PEG10 expresses two reading frames by high efficiency -1 Frameshifting in embryonic-associated tissues," Journal of Biological Chemistry, vol. 282, no. 52, pp. 37359-37369, 2007. 


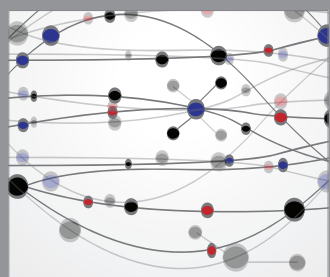

The Scientific World Journal
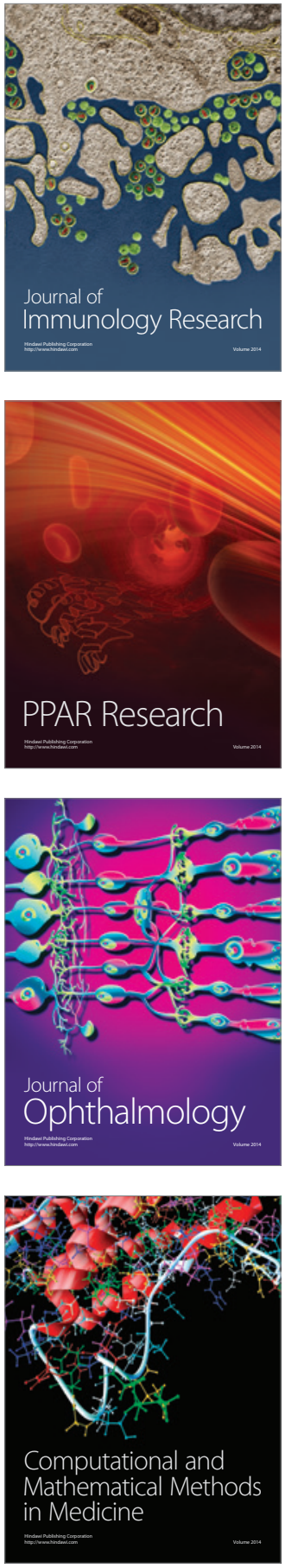

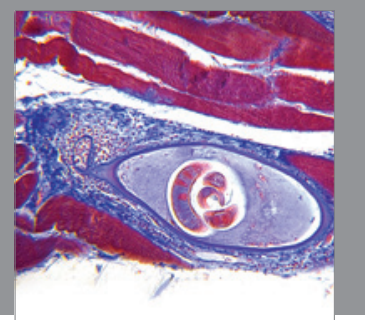

Gastroenterology

Research and Practice
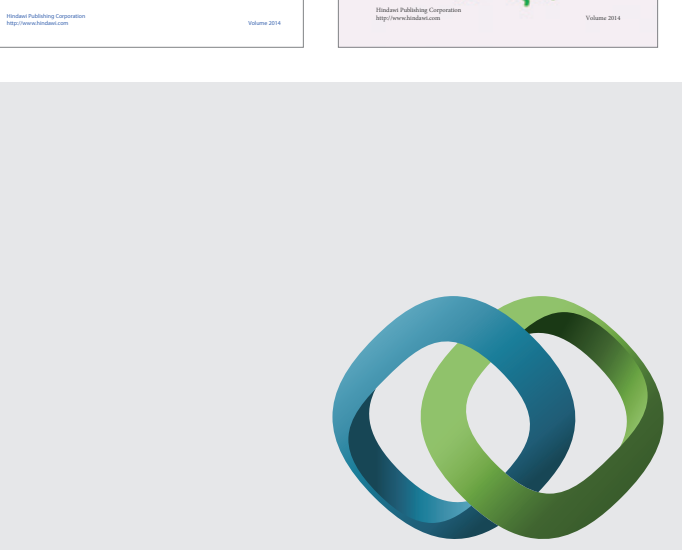

\section{Hindawi}

Submit your manuscripts at

http://www.hindawi.com
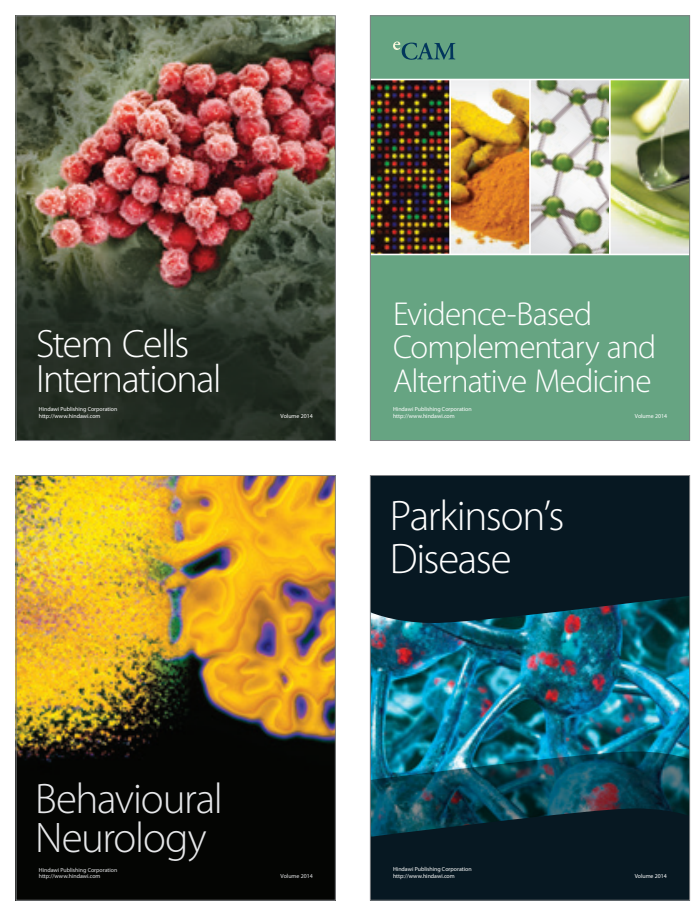

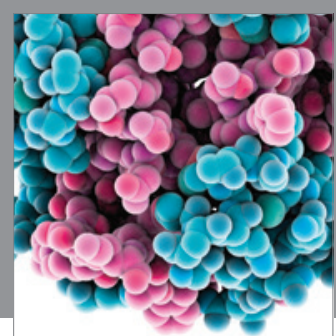

Journal of
Diabetes Research

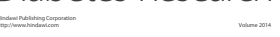

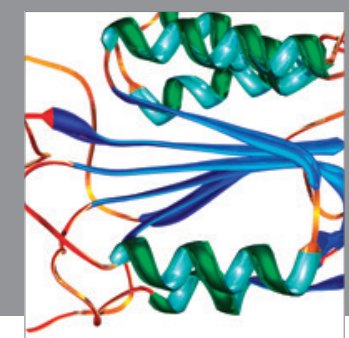

Disease Markers
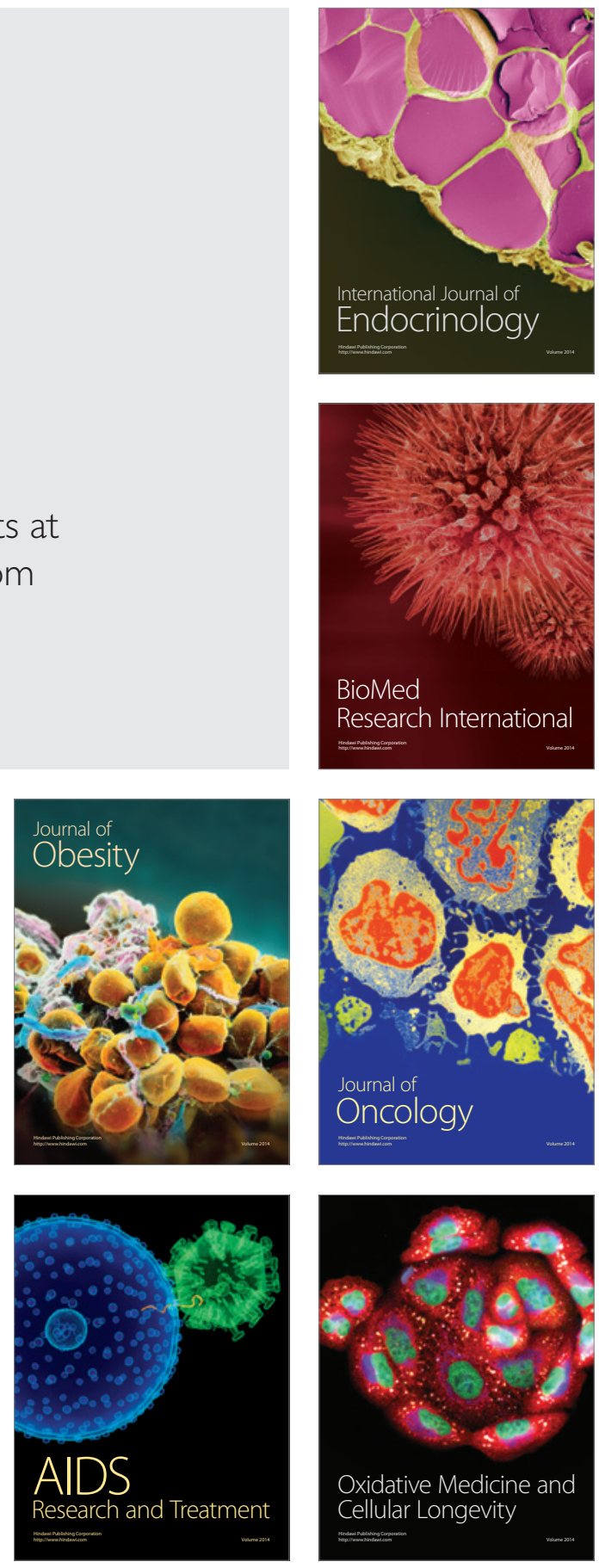